$B$ tubules, which are not hypoplastic and not normally found in adults.

The methods described here have been used to examine testicular tissue from ninety patients with aspermia or oligospermia due to various testicular disorders, but with a normal 46, XY chromosome complement. No tubules were found to contain cells similar to those in type $B$ tubules (unpublished results of J. Philip and myself).

Frøland found two types of tubules during a study of the sex chromatin of patients with Klinefelter's syndrome $^{11}$. In some cases Sertoli cells were sex chromatin positive and in others they were negative. Further work may show whether these findings are related to the observations described here.

I thank Dr Svend G. Johnsen for biospies and useful discussion; Dr Henning Pedersen for biopsies; Dr A. Frøland and Dr J. Philip for carrying out chromosome analyses and Professor G. Teilum for useful discussions and for reading the manuscript of this communication.

Cytogenetic Laboratory,

N. E. SKAKKEB AשK

Department of Obstetrics and Gynaecology A-I,

Rigshospitalet,

University Hospital,

Copenhagen.

Received May 2; revised May 29, 1969.

${ }^{1}$ Overzier, C., in Die Intersexualität (edit. by Overzier, C.), 283 (Georg Thieme, Stuttgart, 1961).

${ }^{2}$ Bunge, R. G., and Bradbury, J. T., J. Crol., 76, 758 (1956).

${ }^{3}$ Grumbach, H. M., Blanc, W. R., and Engle, E. T., J. Clin. Endocrinol., 17, 703 (1957).

"Witschi, E., Nelson, W. O., and Segal, S. J., J. Clin. Endocrinol., 17, 737 (1957).

${ }^{5}$ Ferguson-Smith, M. A., and Munro, I. B., Scot. Med. J., 3, 39 (1958),

${ }^{8}$ Steinberger, E., Smith, K. D., and Perloff, W. H., J. Clin. Endocrinol., 25, 1325 (1965).

${ }^{7}$ Futterweit, W., Fertil. Steril., 18, 492 (1967).

${ }^{8}$ Skakkebæk, N. E., Philip, J., and Hammen, R., Nature, 221, 1075 (1969).

- De la Balze, F. A., Arrillaga, F. C., Irazu, J., and Mancini, R. E., J. Clin. Endocrinol., 12, 1426 (1952).

${ }^{10}$ Johnsen, S. G., Acta Endocrinol., Suppl., 124, 17 (1967).

${ }^{11}$ Froland, A., thesis, Univ. Copenhagen (in the press).

\section{Force, Pain and Electrode Size in the Electrical Stimulation of Leg Muscles}

In patients with paralysed limbs, the existing musculature might be used as the motor drive if suitably programmed stimuli were applied directly to the muscles or to the motor nerves. Our particular concern is with this problem and with the development of devices to make possible the locomotion of patients with totally paralysed lower limbs.

This communication deals with the effects of surface stimulation on normal human beings. The relationships between isometric force, pain and surface area of electrodes have been studied for stimuli which elicit tetanic contractions. Pain studies of various kinds have been performed in the past, and one study on the upper extremities ${ }^{1}$ covered the stimulating waveforms usually encountered in practice. We have used electrodes of various areas, and stimuli consisting of square wave voltage pulses of $50 \mathrm{~Hz}, 0.2 \mathrm{~ms}$ wide and going negative at the stimulus site. These pulses were applied in trains lasting for $1 \mathrm{~s}$ with an intervening rest period also of $1 \mathrm{~s}$. This regimen corresponds roughly to the periods of activity of the various muscle groups in an average walking cycle ${ }^{2}$.

Tibialis anterior and gastrocnemius muscles were studied in some detail and the quadriceps group gave particularly interesting results. In each case electrodes with surface areas of $1,2,4$ and 8 square inches were successively applied over the motor point of the selected muscle. The 12 square inch anodal electrode was placed over the distal end of the stimulated muscle. All the electrodes used were made from a fine stainless steel mesh the interstices of which were filled with 'Redux' electrode paste which was also rubbed into the skin at the sites of application.

For practically isometric contractions, the muscle forces generated by gastrocnemius and tibialis anterior were transduced using a strain-gauge attached to a wooden sole plate to which the foot was firmly strapped. The sole plate was attached to a rigid frame so that the axis of rotation closely coincided with the axis of the ankle joint, and the force was measured 6.5 inches from the axis of rotation, at a point corresponding approximately to the ball of the foot. Force, stimulus voltage and current were measured simultaneously.

Stimulus levels were increased over a period of a few minutes until the subject indicated that he could just tolerate the level for 3-5 $\mathrm{min}$ and then the necessary readings were taken. Eleven male subjects were tested and in all cases the chief determinant of pain seemed to be the total peak current, independent of electrode size. Peak currents of 40 to $105 \mathrm{~mA}$ were supplied and peak voltages were in the range 15 to $40 \mathrm{~V}$.

For the tibialis anterior there was usually an electrode size at which a maximum force of 1.5 to $4.2 \mathrm{~kg}$ occurred. For the gastrocnemius the same effect was noted for more than half the subjects tested, while for the rest a rather larger electrode might have produced larger forces. Maximum forces elicited from gastrocnemius varied from $2 \cdot 1$ to $13 \cdot 7 \mathrm{~kg}$.

There is a limitation on the greatest electrode area that can be used, particularly in the case of the tibialis anterior, when the antagonist muscle group can be stimulated simultaneously by the field spread of stimulating current. It is difficult to compare precisely the various forces produced by electrostimulation with those obtained when the subject volitionally tried to isolate and drive the same muscle groups. But it seems reasonable to conclude tentatively that electrical stimulation can maximally evoke about 40 per cent of the maximum volitional force. The dependence of pain on peak current alone, for the electrode sizes used, may be explained by the fact that to cover a larger area of skin is to embrace more pain sensors which can be stimulated at low current densities. It is worth noting that most subjects preferred stimulating electrodes of at least 2 square inches.

Pilot experiments carried out on the quadriceps group revealed that in three of the four subjects tested it was possible consistently to generate substantial forces (leg weight plus more than $10 \mathrm{~kg}$ ) at the ankle with the leg lying horizontally, and with the knee joint at $90^{\circ}$ when using two carefully placed stimulating electrodes. When active, each electrode was about $30 \mathrm{~V}$ negative with respect to the indifferent electrode and it seemed to be necessary to maintain a small difference in potential between the two active electrodes.

The musculature near the iliotibial band contracted together with the quadriceps per se, and it seemed that, by the combined influence of some mechanical restraint and the applied potentials, the electric field around the motor point of the quadriceps ensured consistent and stable responses to electrostimulation.

\section{MORRIS MILNER}

A. O. Quanbury

Control Systems Laboratory,

Division of Mechanical Engineering,

National Research Council,

Ottawa.

Department of Anatomy,

Queen's University,

Kingston, Ontario.

Received April 17; revised May 16, 1969.

1 Vodovnik, .., Long, C., Regenos, E., and Lippay, A., Arch. Phys. Med. and Rehab., 187 (February 1965).

'Battye, C. K., and Joseph, J., Med. and Biol. Eng., 4, 125 (1966). 